\title{
Avoidance of gluten in children with non-celiac gluten sensitivity is debatable
}

\author{
*Corresponding Author: Chandra Sekhar Devulapalli \\ Norwegian Labour and Welfare Administration (NAV), \\ Medical Advisor Service, Work and Benefits Kristiania, Oslo \\ NO-0653, Norway.
}

Tel: 0047-4060 9823; Email: chandev@gmail.com

Received: Jul 27, 2021

Accepted: Aug 19, 2021

Published: Aug 26, 2021

Archived: www.jjgastro.com

Copyright: (C) Devulapalli CS (2021).

Keywords: gluten; gluten-sensitive enteropathy; gluten-free diet.

\section{Introduction}

It seems that a significant proportion of people adopt a Gluten-Free Diet (GFD) without a medical need. These individuals have either experienced health benefits or they believe that that gluten intake has had a negative effect on them [1]. By all accounts the large proportion of patients are self-diagnosed and start a gluten-free diet without adequate grounds [1]. In addition, some parents use GFD for their children, as they believe that consuming gluten is detrimental to their health [1]. This is reflected by significant increases in the consumption of gluten-free foods in the United States over the last three decades, disproportionate to the prevalence of gluten-related disorders [2]. Evidence is mounting that medicine is harming healthy people through ever earlier detection and ever wider definition of disease [3]. Widening disease definitions mean people at ever lower risks receive permanent medical labels and lifelong treatments that will fail to benefit many of them [4].

\section{Non-celiac gluten sensitivity}

The true prevalence of Non-Celiac Gluten Sensitivity (NCGS) in children is difficult to determine [5,6], however it is estimated to be under 6\% [6]. European guidelines recommend a full investigation, including a duodenal biopsy, in order to rule out celiac disease and wheat allergy while the patient is on a gluten-containing diet, before assessing NCGS $[5,7]$. Definitive diagnosis usually requires observed double-blind, placebocontrolled provocation testing $[8,9]$. Despite this, a number of children are believed to be unnecessarily put on elimination diets without adequate investigation taking place. It is plausible that pediatric patients are often misdiagnosed as having NCGS 
Citation: Devulapalli CS. Avoidance of gluten in children with non-celiac gluten sensitivity is debatable. Japanese J Gastroenterol Res. 2021; 1(3): 1014.

$[1,5,10]$. Adding to the complexity, there is increased marketing of food-specific IgG testing to the general public in recent years, purportedly as a simple way of identifying food intolerances including NCGS $[1,10]$. Uncritical and inappropriate use of such tests increases the likelihood of false diagnoses, resulting in unnecessary dietary restrictions $[1,10]$. Misdiagnosis and false expectations will fuel market demand for gluten-free food products and can be fomented by misleading advertising.

\section{Is gluten avoidance beneficial?}

Gluten-free eating patterns are frequently perceived to be healthier than gluten-containing ones, and GFD has emerged as a lifestyle statement in recent years [6]. However, subjective feelings of improvement after GFD does not indicate it to be healthier than gluten-containing food items [6]. In those with self-reported NCGS, there is currently little evidence of gluten-specific induction of symptoms in randomised blinded rechallenges. The rate and severity of symptoms associated with ingestion of placebo have been overall similar to those following ingestion of gluten in several randomised controlled trials $[1,6,11]$. It seems that symptoms are often wrongly attributed to dietary gluten $[1,11]$.

A recent study demonstrated that gluten-free eating patterns do not have healthier macronutrient or micronutrient profiles, apart from lower sodium levels [12]. On the contrary, gluten-free foods are less optimal for dietary fiber, folate, total protein, vitamin E, magnesium, and potassium [12]. Moreover, in the absence of medical indications to restrict gluten consumption, the potential for lower fiber and several limiting micronutrients highlights the potential for negative effects of gluten-free dietary patterns on long-term health $[6,12]$. It is therefore assumed that children with NCGS who are on GFD will lead to physical effects in the form of nutritional deficiencies.

Based on the current knowledge, it is debatable whether GFD is appropriate in children with NCGS. Currently, the role of gluten is controversial in patients with self-reported NCGS, and gastrointestinal symptoms are often wrongly attributed to dietary gluten. Future clinical research should address and determine more precisely the role of gluten and other nutrients that are involved in the pathogenesis of NCGS. Until that time, it seems appropriate to avoid GFD in children with NCGS as it might lead to nutritional deficiencies in addition to unnecessary expenses for the patient.

\section{References}

1. Devulapalli CS. Gluten-free diet in children - A fad or necessity? Arch Dis Child. 2021; 106: 628-629.

2. Kim HS, Patel KG, Orosz E, et al. Time trends in the prevalence of celiac disease and gluten-free diet in the US population: Results from the National health and nutrition examination surveys 2009-2014. JAMA Intern Med. 2016; 176: 1716-1717.

3. Moynihan R, Doust J, Henry D. Preventing overdiagnosis: how to stop harming the healthy. BMJ. 2012; 344: e3502.

4. Moynihan R, Heath I, Henry D. Selling sickness: the pharmaceutical industry and disease-mongering. BMJ. 2002; 324: 886-91.

5. Devulapalli CS. Non-Coeliac Gluten Sensitivity in children. Tidsskr Nor Laegeforen. 2020 Jan 20;140(2).

6. Devulapalli CS. Non-Celiac Gluten Sensitivity in Children: Controversial Role of Gluten and Diagnostic Enigma. OBM Hepatology and Gastroenterology 2021; 5: 8.

7. Al-Toma A, Volta $U$, Auricchio R et al. European Society for the Study of Coeliac Disease (ESsCD) guideline for coeliac disease and other gluten-related disorders. United European Gastroenterol J. 2019; 7: 583-613.

8. Devulapalli CS. The gluten challenge is still the best option for children with a difficult diagnosis like non-coeliac gluten sensitivity. Acta Paediatr. 2021; 110: 1104-1106.

9. Catassi C, Elli L, Bonaz B et al. Diagnosis of Non-Celiac Gluten Sensitivity (NCGS): The Salerno Experts' Criteria. Nutrients. 2015; 7: 4966-77.

10. Devulapalli CS. Food intolerance in children. Tidsskr Nor Laegeforen. 2020; 140.

11. Gibson PR. Editorial: Inaccuracies in attribution of symptoms due to gluten-not just in those with self-reported noncoeliac gluten sensitivity. Aliment Pharmacol Ther. 2020; 51: 402-403.

12. Taetzsch A, Das SK, Brown C, et al. Are gluten-free diets more nutritious?. An evaluation of self-selected and recommended gluten-free and gluten-containing dietary patterns. Nutrients. 2018; 10: 1881. 